\title{
The Clinical Analysis on Acute Oral Cavity Mucositis Caused by the Radiotherapy for the Head and Neck Cancer
}

\author{
Hongying Lin ${ }^{1, \mathrm{a}}$, Min $\mathrm{Ma}^{1}{ }^{1}$, Shengcun Zhang ${ }^{1}$ \\ ${ }^{1}$ Department of Cancer and Hematology, The third hospital of Jinan ,Jinan, 250132,China \\ alilin788@126.com \\ *Corresponding author mamin@126.com
}

\begin{abstract}
Keywords: Reduced glutathione injection; Head and neck cancer; Radiation therapy; Acute inflammation of the oral mucosa
\end{abstract}

\begin{abstract}
Objective: To observe and discuss the clinical effect of the treatment of the reduced glutathione injection acute oral cavity mucositis caused by radiotherapy for head and neck cancer. Methods: We select from August 2012 to August 201478 cases of the head and neck radiotherapy of head and neck cancer patients, both by pathology diagnosed with head and neck malignant tumours. The patients were randomly divided into treatment group and control group, two groups of the dose of radiation technology and basic consistent. The treatment group is used when patients mucositis II level, intravenous drip, and the reduced glutathione injection was given until the end of radiotherapy. The control group when patients mucositis II level, began to give intravenous drip $0.9 \%$ saline until the end of radiotherapy. Results: For the treatment of oral cavity mucous membrane inflammation, two groups of patients with oral cavity mucositis evaluation result comparison, are no obvious difference, there is no statistical significance $(\mathrm{P}>0.05)$. After radiotherapy, the inflammation of the oral mucosa in patients with two groups of case investigation and comparison results show that the evaluation results of patients with oral mucositis treatment group was obviously better than the control group, the significant difference was statistically significant $(\mathrm{P}<0.05)$. Conclusion: The reduced glutathione injection for treating head and neck radiotherapy caused by acute inflammation of the oral mucosa has the certain effect, and it is worthy of clinical using.
\end{abstract}

\section{Introduction}

The Acute inflammation of the oral mucosa for oral mucosa of normal colour, shape a part, such as integrity and function change of disease. Main symptoms of oral mucosa hyperaemia, erythematic, erosion, ulcer bleeding, severe cases can cause infection. Acute inflammation of the oral mucosa is the most common acute complications of radiotherapy for head and neck cancer, more than $2 \sim 3$ weeks after radiation therapy, the patient to bring huge pain and sufferings, affect patients eat, serious refusing food drink, resulting in patients with electrolyte disorder, and even forced to discontinue treatment, make it become a problem urgently to be solved in clinical radiotherapy for head and neck cancer.

\section{Materials and Methods}

\section{Materials}

We choose 78 cases of head and neck radiotherapy of head and neck cancer patients from August 2012 to August 2014, both by pathology diagnosed with head and neck malignant tumours. Patients were randomly divided into treatment group and control group, treatment group (39 cases, male 22 cases, female 17 cases; Aged 22 to 75 years, mean age (46.5 \pm 18.9$) ; 22$ cases of nasopharyngeal carcinoma, laryngeal cancer 12 cases, 5 cases of the parotid gland carcinoma. 23 cases of control group 39 cases, male, female 16 cases; 23 74 years old, average age $(45.8 \pm 19.2)$; 23 cases of nasopharyngeal carcinoma, laryngeal cancer 12 cases, 4 cases of parotid gland carcinoma. Two groups of patients with are no significant differences in age, sex and disease, comparable. 


\section{Methods}

After radiotherapy, when the evaluation for complications in patients with oral mucositis II level, treatment group: Rio the reduced glutathione injection, usage and dosage: $1.8 \mathrm{~g}$ one day, 1, 1) dissolved in $250 \mathrm{ml}$ of $0.9 \%$ saline intravenous drip, until the end of radiotherapy. In the control group: $250 \mathrm{ml} 0.9 \%$ saline intravenous drip, 1, 1, until the end of radiotherapy, the statistical analysis before and after treatment in patients with oral cavity mucositis.

\section{The evaluation standard}

It refers to the WHO classification standard oral mucositis, oral mucosa reaction can be divided into 5 levels; First, I, without exception of the oral mucosa; Second, II, oral cavity mucous membrane appears erythematic, pain; Third, III, occurrence erythematic, ulcers of the oral mucosa, can eat; Fourth, IV, oral mucosa ulcer, can into the liquid diet; V, oral mucosa ulcer, can't eat.

\section{Statistical processing}

With mathematical statistics software SPSS19.0 for data collection and statistical analysis, and chi-square test, $\mathrm{P}<0.05$, significant difference has statistical significance.

\section{Results}

For oral cavity mucous membrane inflammation before the treatment, two groups of patients with oral cavity mucositis evaluation result comparison, no obvious difference, there is no statistical significance $(\mathrm{P}>0.05)$. After radiotherapy, inflammation of the oral mucosa in patients with two groups of case investigation and comparison results show that the evaluation results of patients with oral mucositis treatment group was obviously better than the control group, significant difference is statistically significant $(\mathrm{P}<0.05)$.

Table 1: The compare of two groups of patients with oral cavity mucositis evaluation results

\begin{tabular}{ccccccc}
\hline Group & $\mathrm{n}$ & $\begin{array}{c}\text { Level } \\
\mathrm{I}\end{array}$ & Level II & Level III & Level IV & Level V \\
\hline $\begin{array}{c}\text { The treatment } \\
\text { group }\end{array}$ & 39 & $2(5.13)$ & $22(56.41)$ & $12(30.77)$ & $2(5.13)$ & $1 \quad(2.56)$ \\
$\begin{array}{c}\text { The control } \\
\text { group }\end{array}$ & 39 & $0(0)$ & $7(17.95)$ & $17(43.59)$ & $10(25.64)$ & $5(12.82)$ \\
X 2 & & 6.043 & 12.327 & 7.895 & 9.216 & $\begin{array}{l}\text { (1).426 } \\
\text { P value }\end{array}$ \\
\hline
\end{tabular}

\section{Discussion}

The cancer radiotherapy, the irradiation not only the primary lesions, but also will damage the normal tissue. The radiation effects on the body make the moisture by excitation and ionization produces a lot of strong toxicity of free radicals, lead to biological molecules bond rupture, molecular structure destruction and cell death. For head and neck radiotherapy, after exposure of the oral mucosa epithelial damage congestion, erosion, ulcer bleeding, product increases with the increased amount of radiation ulcer, pain intensified, the serious influence the patient's quality of life, and even affect the treatment. The main damage mechanism and ionizing radiation is effects on oxygen free radicals of the oral cavity.

\section{The acute inflammation of the oral mucosa}

It is the most common adverse reactions in head and neck radiotherapy of radioactive oral cavity mucous membrane inflammation, also known as the radiation induced oral mucositis (RTOM). Because of the direct and indirect radiation damage, radiotherapy with chemotherapy, patients with 
tumor in the special environment and mental function, make the head and neck radiotherapy in patients with higher incidence of oral cavity mucous membrane inflammation, its incidence in patients with head and neck radiotherapy were $85 \% \sim 100 \%$. The sensitivity of the epithelial cells of radiation is stronger, after about $20 \mathrm{~Gy}$ ensued mucosal inflammation, when treatment dose up to $30 \sim 40$ Gy inflammation can be affected all of the oral mucosa, appear diffuse erosions, hyperemia, clinical manifestation is: reduce salivation, dry mouth, diffuse inflammation of the oral mucosa, pseudo membrane formation, or pharynx and ulcer pain, peak between 14 and 21 days. Or pharyngeal pain often makes patients eat couldn't even eat, resulting in the lack of nutrition, water and electrolyte balance disorder, even with patients with low immunity, easily become infected entrance, thus affecting the radiotherapy plan implementation and the effect of tumor treatment. So the correct oral care is important in patients with head and neck radiotherapy, radiotherapy in our families take mouth ice after radiotherapy to prevent oral cavity mucous membrane inflammation, in reducing the oral pain, reduce the incidence, alleviate the severity of the reaction, to delay its occurrence time, certain achievements have been made in the delay its progress.

\section{The happening time of the acute inflammation of the oral mucosa}

Oral mucositis caused by radiotherapy the main location for the front of the lips, cheeks, the soft palate, tongue and mouth floor and so on non keratinized mucosa, and gums, tongue back or hard palate is rarely, if ever, it may be associated with the proliferation rate of the cells. And RTOM degree and the duration and source and cumulative dose of irradiation dose intensity, the volume of a radiation mucous membrane, smoking, drinking habits, and other factors such as dry mouth or oral infections. RTOM radiotherapy in more 3 weeks, until the end of radiotherapy and lasted longer. According to the research pointed out that the radiation mucositis pain caused by radiotherapy in more, 2,3 weeks to 5 weeks of severe pain, until the end of radiotherapy. In the standard of conventional segmentation irradiation (200 cGyPd), mucosal erythema occur in within the first week of treatment, radiation treatment caused by scattered or fusion in the peak of mucous membrane inflammation for $4 \sim 5$ weeks of treatment, daily radiation dose is less than 200 cGy cause mucosal inflammatory reaction is lighter, however, in the accelerated radiotherapy, mucosal inflammation peak within 3 weeks. Radiation damage in connective tissue to further enhance the effect of epithelial cells, in the host immune activity, oral cavity mucous membrane inflammation is usually caused by radiotherapy in stop light within 3 weeks to heal. Mucosal inflammation caused by insert irradiation graft usually appears in $7 \sim 10 \mathrm{~d}$, reached its peak within 2 weeks. This kind of injury is often in a few weeks to heal, unless there is a large areas of mucosa damage.

\section{The pathogenesis of the acute inflammation of the oral mucosa}

RTOM pathogenesis is not fully clear; most scholars think RTOM is the result of joint action of many factors. Common damage mechanism has the following kinds:

(1) The pathological factors

Malignant tumor patients physiological function disorder, the body resistance is poor, and poor oral hygiene, easy to breed bacteria in oral cavity, not only increases in the number, and given priority to with anaerobic bacteria and spoilage organisms. Bacteria break down sugars, enhanced to produce acid fermentation, leads to the formation of a local inflammatory ulcer. Think now the basic pathology including direct damage mechanism and indirect damage mechanism. Direct damage mechanism for radiotherapy in radiation cause oral cavity mucous membrane directly reduces the number of cells. According to the relevant research has shown that patients before radiotherapy for head and neck cancer of the oral mucosa cells number about 1000 / was separated with conventional irradiation $2 \mathrm{~Gy} / \mathrm{d}$, five times a week, cell count dropped to 500 after treatment 1 w/was, then due to the compensatory function of the oral mucosa and the establishment of, cells reduce speed slowed significantly, when the end of radiotherapy (about $7 \mathrm{w}$ ) of the oral mucosa cells number about 400 / was. Indirect damage mechanism of oral mucosa damage is due to the release of inflammatory mediators, radiation or ant tumor drugs cost plays a protective role of saliva. Neutrogena caused by radiation therapy at the same time also promoted the development of RTOM, also promote the bacteria, fungi and viruses on the damage of mucous membrane planting and 
breeding, and neutrophil count associated with the development of oral mucositis, neutrophil recovery and there is a linear relationship improvement of inflammation of radioactive oral cavity, serious radioactive stomatitis may occur in patients with bone marrow cells lack.

(2) The treatment factors

1) The radiation damage basal cell, make mucous membrane cell division of compensation mechanism is affected, the thickness of the membrane is reduced, increased brittleness, eating tiny mechanical stimulation to mucous membrane damage increased chance; Head and neck tumor itself can cause changes in the saliva of some ingredients; Radiotherapy and directly undermine reduce the production of saliva, salivary glands which changes oral bacteria oral micro structure and ecological environment, according to the report after radiotherapy Llory and Dammron acid bacteria and radiation significantly increased significantly, saliva buffering capacity decline. Salivary glands with radioactive damage, inhibition, saliva flow and quality are greatly reduced, oral self-cleaning function and immune function decreased significantly, leading to poor oral hygiene, acidity, $\mathrm{pH}$, disorder of flora balance, cause mucosal inflammation, burst; Patients often have swelling, dry mouth, mouth pharynx mucosa and oral ulcer. 2) In some patients with radiation and chemotherapy combined with, due to the chemotherapy drug to inhibit or kill tumor cells at the same time, to update faster normal tissues such as oral mucosal epithelium of the toxicity, the drug was so happen quickly oral mucosa atrophy, thinning, increased brittleness and inflammation, pain, ulcer.

(3) The internal environment factors

Malignant tumor patients with systemic and comprehensive treatment of the dual mechanism, which can cause micro ecological imbalance, environment in oral cavity ptar reported bacterial change, including streptococcus mutans, lactic acid bacteria, candida (mainly candida albicans) and staphylococcus aureus bacteria produce acid significantly increased, accompanied by blood streptococcus, neisseria bacteria, and clostridium bacteria than the number of bacteria produce acid. Associated with the application of broad-spectrum antibiotics, antibiotics in the body to normal bacteria to kill or inhibit stranger, antagonism between bacteria between disappear, candida albicans to multiply, easily lead to infections of double or multiple. Other produce vitamin kill or inhibit bacteria are, cause vitamin deficiency, mucosal immunity decline and produce inflammation of the oral mucosa. After radiotherapy of saliva decrease, often appear acid product gathered themselves together, and $\mathrm{pH}$ value lower than normal, lead to inflammation of the oral mucosa.

(4) The mental factors

Malignant tumor patients due to the influence of social environment, coupled with treatment time is long, the side effects of radiotherapy, the patient's psychological pressure big, cause mental tension, insomnia, poor appetite, resistance to drop, can cause inflammation of the oral mucosa.

\section{The clinical analysis of the reduced glutathione injection treatment acute oral cavity mucositis caused by radiotherapy for head and neck cancer}

The reduced glutathione injection, also known as glutathione, is a kind of three human natural synthesis in the cytoplasm of peptide, composed of glutamate, glycine and cysteine residues of containing sulphur ( $\mathrm{SH}$ ), the activation of REDOX system in the body, activate the $\mathrm{SH}$ enzyme, detoxification and other important role, and participates in the metabolism of a variety of important biochemical reactions, with electrophonic, oxygen free radicals and other toxic substances, with a wide range of antioxidant effect, protect the cells.

For stratified squamous epithelium of the oral mucosa, and update faster, have higher sensitivity to radiation. Mucous membrane of the acute radiation reaction is caused by the radiation mucous membrane base layer cell mitosis of death, main show is erythema, membrane, erosion and ulcer, etc., the main damage mechanism and ionizing radiation effect on oxygen free radicals of the oral cavity. Oxygen free radicals and cell membrane multivalent unsaturated fatty acids, lead to imbalance of cell membrane multivalent unsaturated fatty acid and protein, the damage of membrane receptor on the surface of the function, form a new ion channels, the inflow, resulting in a large number of $\mathrm{Ca} 2+$, mitochondria and lysosome damage and cell death; And oxygen free 
radical effect on containing sulphur amino acid protein denaturation and inactivation of enzyme, interstitial oxygen free radicals can destroy epithelium of hyaluronic acid and collagen fibres, aggravating mucosa damage.

The reduced glutathione injection is a kind of natural synthetic peptide in the human cytoplasm, composed of glutamic acid, cysteine and glycine, containing sulphur $(\mathrm{SH})$, widely distributed in the body's organs, in order to maintain cell biological function plays an important role. The reduced glutathione is the prosthetic group of glyceraldehydes phosphoric acid dehydrogenase, and glyoxalase and triose dehydrogenase coenzyme, participate in the Krebs cycle and glucose metabolism in the body, it can activate a variety of enzyme [such as sulphur (SH) enzyme], so as to promote the sugar, fat and protein metabolism, and can influence the process of cell metabolism. The reduced glutathione combining thiol and free radicals in the body can be converted to easy metabolic acids material so as to accelerate the elimination of the free radicals, and protect the normal cells..

\section{Conclusion}

The main purpose of this study is to observe the prevention and treatment of acute inflammation of the oral mucosa, the results show that preventive drug (radiotherapy 1 day) and therapeutic drug (grade II mucosal inflammation began) using the reduced glutathione injection can significantly reduce the radiotherapy for head and neck cancer caused by the incidence of acute inflammation of the oral mucosa, beat the degree of pain; Preventive medicine and reduce the incidence of grade III or IV mucositis, more conducive to improve the quality of life of patients during treatment. Therefore, preventive use of the reduced glutathione injection may be of more significance for prevention and control of mucous membrane inflammation. The reduced glutathione injection best delivery time in every time before radiotherapy for medicine or after radiotherapy for medicine, there is no comparison, needs further research.

\section{Acknowledgement}

This research was financially supported by the National Science Foundation.

\section{References}

[1] Wagner W, Alfrink M, Haus U, et al. Treatment of irradiation-induced mucositis with growth factors(rhGM-CSF)in patients with head and neck cancer. Anticancer Research . 2012(1).

[2] Amit Deorukhkar , Sunil Krishnan. Targeting inflammatory pathways for tumor radiosensitization[J]. Biochemical Pharmacology. 2012 (12)

[3] David I. Rosenthal, Andrea Trotti. Strategies for Managing Radiation-Induced Mucositis in Head and Neck Cancer[J]. Seminars in Radiation Oncology. 2013(1)

[4] Maria Luisa P. Melo, Gerly A. C. Brito, Rudy C. Soares, Sarah B. L. M. Carvalho, Johan V. Silva, Pedro M. G. Soares, Mariana L. Vale, Marcellus H. L. P. Souza, Fernando Q. Cunha, Ronaldo A. Ribeiro. Role of cytokines (TNF- $\alpha$, IL-1 $\beta$ and KC) in the pathogenesis of CPT-11-induced intestinal mucositis in mice: effect of pentoxifylline and thalidomide[J]. Cancer Chemotherapy and Pharmacology. 2013(5)

[5] Monica Fliedner, Brigitte Baguet, Joachim Blankart, Michelle Davies, Elisabete Henriques, Angela Leather, Ewa Mazur, Katalin Mihály, Liesbet Peeters, Agnes Radványiné, Blanka Sedlackova. Palifermin for patients with haematological malignancies: Shifting nursing practice from symptom relief to prevention of oral mucositis[J]. European Journal of Oncology Nursing . 2012(06)

[6] Jun-lin Yi, Li Gao, Xiao-dong Huang, Su-yan Li, Jin-wei Luo, Wei-ming Cai, Jian-ping Xiao, Guo-zhen $\mathrm{Xu}$. Nasopharyngeal carcinoma treated by radical radiotherapy alone: Ten-year experience of a single institution[J]. International Journal of Radiation Oncology, Biology, Physics. $2012(1)$ 
[7] A. Ahamad, L. Dong, L. Zhang, K. Ang, J. O' Daniel,L. Theriot, J. Asper, M. Chambers, S. Palla, A. Garden. 181[J]. International Journal of Radiation Oncology, Biology, Physics. 2012 (3) [8] Montserrat Vera - Llonch, Gerry Oster, May Hagiwara, Stephen Sonis. Oral mucositis in patients undergoing radiation treatment for head and neck carcinoma[J]. Cancer. 2013 (2)

[9] F. Cianfarani, R. Tommasi, C. M. Failla, M. T. Viviano, G. Annessi, M. Papi, G. Zambruno, T. Odorisio. Granulocyte/macrophage colony - stimulating factor treatment of human chronic ulcers promotes angiogenesis associated with de novo vascular endothelial growth factor transcription in the ulcer bed[J]. British Journal of Dermatology. 2012 (1)

[10] Giuseppe Masucci, Peter Broman, Charles Kelly, Sten Lindahl, Lena Malmberg, Johan Reizenstein, Martin Ålenius, Rolf Lewensohn. Therapeutic efficacy by recombinant human granulocyte/monocyte-colony stimulating factor on mucositis occurring in patients with oral and oropharynx tumors treated with curative radiotherapy[J]. Medical Oncology. 2013 (3). 